\title{
Measurement of helium isotope ratio using multi-turn time-of-flight mass spectrometer
}

\author{
Y. HATTORI ${ }^{1 *}$, Y. AKIYAMA $^{2}$, AND H. SUMINO ${ }^{3}$ \\ ${ }^{1}$ Dept. Basic Sci., Grad. Sch. Arts \& Sci., Univ. Tokyo, \\ Tokyo 153-8902, Japan (*correspondence: \\ yukihatt2525@g.ecc.u-tokyo.ac.jp)
}

The helium isotope ratio $\left({ }^{3} \mathrm{He} /{ }^{4} \mathrm{He}\right.$ ratio) shows different values in geochemical reservoirs such as the atmosphere, crust, and mantle. The ${ }^{3} \mathrm{He} /{ }^{4} \mathrm{He}$ ratios of volcanic gases have values between magmatic (up to $1.1 \times 10^{-5}$ or lower) and crustal (less than $1 \times 10^{-7}$ ) values. When magma becomes active, the ${ }^{3} \mathrm{He} /{ }^{4} \mathrm{He}$ ratio of volcanic gas may increase as the contribution of magmatic helium is expected to be higher. ${ }^{3} \mathrm{He} /{ }^{4} \mathrm{He}$ ratio of volcanic gas has a great potential as a monitoring tool of volcanic activity. Continuous analysis of volcanic gas is necessary to monitor volcanic activity; however, it is difficult for ${ }^{3} \mathrm{He} /{ }^{4} \mathrm{He}$ ratio because magnetic-sector mass spectrometer equipped with a massive electromagnet is currently used to analyze helium isotopes for requests of high mass resolution and high sensitivity.

We have been developing a new technique of noble gas analysis using multi-turn time-of-flight mass spectrometer "infiTOF". The high mass resolution achieved by infiTOF is more than enough to distinguish ${ }^{3} \mathrm{He}^{+}$from $\mathrm{HD}^{+}$. However, sensitivity of a normal infiTOF was not high enough to analyze noble gases in volcanic gas because most of noble gas molecules admitted to the infiTOF were pumped out by vacuum pumps directly connected to the spectrometer before ionized. In order to realize static operation (i.e. the spectrometer is isolated from the vacuum pumps so that sample gas molecules are closed off within the spectrometer envelope), we installed valves between the spectrometer volume and the vacuum pumps. Getter pumps, which absorb active gases but not noble gases, were also installed to keep the pressure in the spectrometer low during the operation. In addition, we introduced the ion counting method for signal processing to detect weak signals of ${ }^{3} \mathrm{He}$. As a result, significant number of ${ }^{3} \mathrm{He}$ in the helium standard gas (HESJ ${ }^{[1]}$ ) has been detected. Although as high sensitivity as that of the magnetic-sector mass spectrometer has not yet been achieved, accumulating number of ${ }^{3} \mathrm{He}$ signals with a longer time will increase statistical precision of ${ }^{3} \mathrm{He} /{ }^{4} \mathrm{He}$ ratio enough to distinguish the origin of helium in volcanic gas sample based on its isotope ratio.

[1] Matsuda et. al., Geochem. J. (2002). 